\title{
Prevalência de extubação não planejada e fatores associados em uma unidade de terapia intensiva neonatal
}

\author{
Prevalence of unplanned extubation \\ and associated factors in a neonatal \\ intensive care unit
}

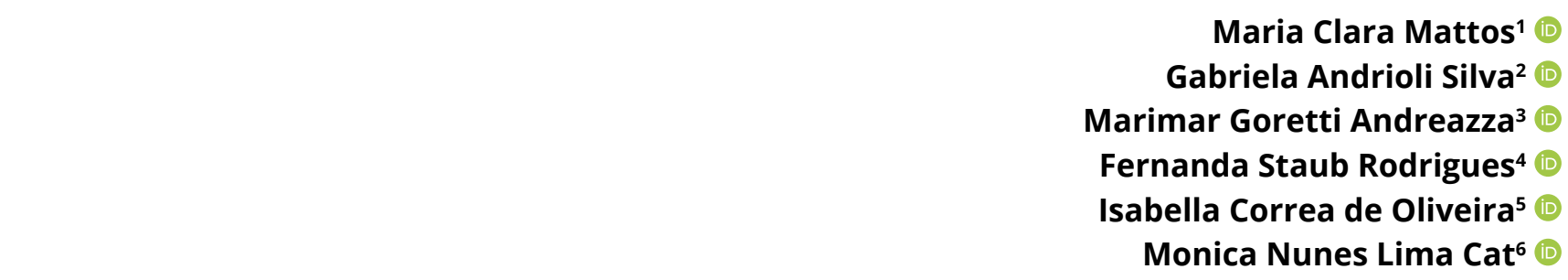

1,2,4-6Universidade Federal do Paraná (Curitiba). Paraná, Brasil. mclaralmattos.94@gmail.com, andrioligabriela@gmail.com, fernandastaubr@gmail.com, isabella.oliveira21@gmail.com, monica.lima.ufpr@gmail.com ${ }^{3}$ Autora para correspondência. Hospital de Clínicas, Universidade Federal do Paraná. Curitiba, Paraná, Brasil. marimar.andreazza@hc.ufpr.br

RESUMO | INTRODUÇÃO: Intubação endotraqueal é procedimento comum nas Unidades de Terapia Intensiva Neonatais e tem como evento adverso a extubação não planejada, um incidente grave, associado a morbidade neonatal. OBJETIVO: Verificar a prevalência de extubação não planejada e fatores associados em recém-nascidos submetidos à ventilação mecânica na unidade de terapia intensiva neonatal por condições respiratórias, cardíacas ou por pós-operatório. MATERIAIS E MÉTODOS: Estudo transversal, retrospectivo realizado entre março a dezembro de 2017. Os dados foram coletados da ficha de notificação do serviço e incluíram: sexo, peso no momento do evento, comorbidades do paciente, data e hora de ocorrência, condições associadas e conduta pós-extubação. RESULTADOS: Em 38 recém-nascidos em ventilação mecânica foram registrados 72 eventos, a prevalência da extubação não planejada de 4,6\%. Entre os fatores identificados, peso inferior a $1.500 \mathrm{~g}$ foi observado em 20 recém-nascidos $(52,6 \%)$ e prematuridade em 25 (65,8\%). Em 15 recém-nascidos (39,5\%) houve mais de um evento/paciente. Peso inferior a 2.500g esteve associado a um risco 6 vezes maior de recorrência do incidente. As condições associadas aos eventos foram agitação motora do recém-nascido (50\%), manuseio da cânula endotraqueal (28,3\%) e durante procedimentos de rotina do recém-nascido $(21,7 \%)$. Reintubação foi necessária em 58 casos (80,5\%), sendo imediata em 20 (34,5\%). CONCLUSÃO: A agitação motora e o manuseio da cânula endotraqueal, portanto, foram os fatores mais associados aos eventos, a recorrência foi mais frequente em recém-nascido com peso inferior a $2.500 \mathrm{~g}$. Medidas de prevenção devem incluir o manejo adequado da agitação motora do recém-nascido e implementação de protocolos de manuseio da cânula endotraqueal.

PALAVRAS-CHAVE: Extubação não planejada. Prematuridade. Segurança do paciente.
ABSTRACT | INTRODUCTION: Endotracheal intubation is a common procedure in Neonatal Intensive Care Unit (NICU) and Unplanned Extubation (UE) is a severe related event, which increases neonatal morbidity. OBJECTIVE: To verify the prevalence of UE and associated factors in newborns submitted to mechanical ventilation (MV). MATERIALS AND METHODS: This is a cross-sectional retrospective study, including 38 newborns and 72 UE. The collected parameters were: gender, weight at the time of extubation, diagnosis, time of UE, associated conditions and conduct. The statistical analysis included the Fisher's exact test and odds ratio (Statistica ${ }^{\circledR}$ ). RESULTS: A total of 72 UE were recorded in 38 newborns, with a rate of 1.561 intubated patients/day and UE prevalence of $4.6 \%$. Weight less than 1,500g was observed in 20 newborns (52.6\%) and prematurity in 25 (65.8\%) and when it was less than $2500 \mathrm{~g}$ it was associated with a 6 -fold increased risk of recurrent UE. In 15 newborns (39.5\%) there was more than one event per patient. The conditions associated with increased UE risk were motor agitation of the newborn (50\%), endotracheal tube (ETT) manipulation (28.3\%) and routine procedures (21.7\%). Reintubation was necessary in 58 cases (80.5\%), being immediate in 20 (34.5\%). CONCLUSION: The prevalence of UE was high and strongly associated with motor agitation and manipulation of ETT. Its recurrence was more frequent in newborns weighing less than 2,500 g. Therefore, prevention measures should include adequate management of the motor agitation in newborns and implementation of protocols for handling the ETT.

Keywords: Unplanned extubation. Prematurity. Patient Safety. 


\section{Introdução}

Recém-nascidos gravemente enfermos rotineiramente necessitam de intubação endotraqueal e ventilação mecânica (VM), procedimento associado a maior sobrevida ${ }^{1}$. Entretanto, esta condição expõe os pacientes à extubação não planejada (ENP), efeito adverso grave e frequente nas UTI neonatais. É considerada ENP toda retirada do tubo endotraqueal, em um momento não planejado, removido acidentalmente pelo paciente ou de forma não intencional pela equipe de saúde², podendo ocasionar graves consequências para a vida do paciente. Dentre as complicações mais frequentes estão a hipoxemia, hipercapnia, atelectasia, pneumotórax, danos na via aérea, alterações hemodinâmicas e necessidade de reintubação em situações urgentes e menos controladas².

Neonatos são considerados uma população de risco para extubação não planejada devido às dificuldades de fixação da cânula endotraqueal (CET), face pequena, curto comprimento da traqueia e utilização de tubos endotraqueais sem balonete ${ }^{1,2}$. Dentre as condições associadas mais comuns encontram-se agitação motora do recém-nascido e procedimentos realizados pelos profissionais de saúde, tais como banho no leito, transporte, troca de fixação da cânula endotraqueal e mudança de decúbito ${ }^{1-4}$. Sabe-se que tempo de ventilação mecânica prolongada ${ }^{5,6}$ e frequência e tempo de manuseio dos recém-nascido pela equipe e pelos pais também são fatores que aumentam o risco $0^{1,2,7,8}$.

Estima-se que a taxa de ENP entre pacientes adultos varia entre 0,10-3,61/100 pacientes intubados/dia, enquanto gira em torno de 0,11-2,70/100 em UTI pediátricas, sendo de 2 a 3 vezes maior em UTI neonatais. A incidência estimada desse evento em recém-nascido nos EUA é de 0,14-6,6/100 e no Brasil, um estudo realizado em Campinas (São Paulo) apontou incidência de 5,34/1005. É $4^{\circ}$ evento adverso mais frequente em UTI neonatais, atrás apenas de erros com medicação, infecções associadas aos cuidados em saúde (IACS) e lesões cutâneas.

O objetivo desta pesquisa foi verificar a prevalência e fatores associados à extubação não planejada em recém-nascidos internados na UTI Neonatal da instituição participante, submetidos à ventilação mecânica por condições respiratórias, cardíacas ou por pós-operatório. E, desse modo, incrementar as ações de vigilância e prevenção deste incidente frequente, contribuindo para as ações de segurança do paciente.

\section{Material e métodos}

Estudo transversal, retrospectivo realizado em uma UTI neonatal de um Hospital Universitário. Foram incluídos os dados de neonatos que necessitaram de intubação endotraqueal e ventilação mecânica no período de março a dezembro de 2017, aqueles que apresentaram eventos de extubação não planejada nesse período. Foram excluídos neonatos que tiveram extubação planejada nesse período. Os dados coletados foram extraídos de um formulário específico para ENP padronizado no serviço, preenchido pela equipe no momento em que ocorreu o evento e continham informações sobre sexo, peso no momento da extubação, comorbidades do paciente, data e horário da extubação não planejada, possíveis condições associadas ao evento e conduta após a ocorrência. Foram excluídos as extubações planejadas. $\mathrm{Na}$ análise estatística foi aplicado o teste exato de Fisher e calculado o odds ratio para estimar a razão de chance de ENP recorrente. Foi utilizado o software Statistica (Statsoft $\AA$ ), considerando o nível de significância de $5 \%$ e poder de teste de $90 \%$. O estudo foi aprovado pelo Comitê de Ética em Pesquisa em Seres Humanos da Instituição, conforme parecer número 2.426.709 (CAAE 79801417100000096). 


\section{Resultados}

A UTI neonatal, do Hospital Universitário, unidade de atendimento de nível terciário com 10 leitos de UTI e 10 de risco intermediário, apresentou, no período de estudo, ocupação de 8.170 pacientes/dia e 125 recém-nascido foram intubados, com taxa de pacientes intubados/dia de 1.561. Em 38 recém-nascido foram registrados 72 eventos de extubação não planejada. Considerando a frequência de pacientes intubados por dia, observou-se prevalência de 4,6/100.

Dos 38 recém-nascidos, 18 eram do sexo masculino $(47,4 \%)$ e 20 do sexo feminino $(52,6 \%)$. Destes, 13 $(34,2 \%)$ apresentavam peso, no momento do evento, inferior a 1.000g, $7(18,5 \%)$ entre 1.000 e $1.500 \mathrm{~g}, 4$ $(10,5 \%)$ entre 1.500 a $2.500 \mathrm{~g}$ e $14(36,8 \%)$ com peso superior a 2.500g. Prematuridade foi observada em $25 \mathrm{ca}$ sos $(65,8 \%)$ e 11 recém-nascidos (28,9\%) apresentavam algum tipo de malformação. Todos estavam sob ventilação mecânica por condições respiratórias, cardíacas ou por pós-operatório. Os recém-nascidos estavam, em sua maioria $(95,0 \%)$, intubados por via orotraqueal e os demais $(5,0 \%)$ por via nasotraqueal. No período de estudo a taxa de pacientes em ventilação mecânica ao mês foi de 13, variando de 7 a 23 dias, (Tabela 1).

Dos 38 neonatos, 23 apresentaram um evento de ENP $(60,5 \%)$ e 15 apresentaram mais de um (39,5\%), destes dois eventos em cinco casos; três em quatro casos; quatro em quatro casos; cinco em apenas 1 caso e 7 eventos em mais 1 caso. Totalizando 72 eventos de extubação não planejada. Excluindo estes dois últimos casos de ENP recorrente, cujos recémnascido apresentavam malformações cervicais que dificultavam a intubação, a prevalência corrigida na unidade seria de 3,8/100.

Em 28 casos houve um ou dois eventos de ENP $(73,6 \%)$, sendo 19 deles $(67,8 \%)$ em recém-nascidos com peso no momento do evento igual ou inferior a 2.500g $(p<0,01)$. O risco de mais de 1 episódio de ENP foi seis vezes maior nestes recém-nascido com peso inferior a 2.500g (OR =6,6; IC 95\% = 1,7-24,8).
Considerando os 72 eventos, 29 (40,3\%) ocorreram em recém-nascido com peso no momento da extubação inferior a 1.000g, 10 (13,9\%) entre 1.000 e $1.500 \mathrm{~g}$, $6(8,3 \%)$ entre $1.500 \mathrm{~g}$ e $2.500 \mathrm{~g}$ e $27(37,5 \%)$ com peso superior a $2.500 \mathrm{~g}$.

Em 60 eventos (83,3\%) foi possível analisar causa da extubação, ocorrendo durante manuseio da cânula endotraqueal (fixação, troca ou reposicionamento) em 17 casos (28,3\%), durante procedimentos realizados por profissionais de saúde (pesagem, passagem de sonda nasogástrica, colocação de drenos, coleta de amostra sanguínea ou realização de exames de imagem) em nove casos (15,0\%), durante realização de exame clínico em quatro casos $(6,7 \%)$ e durante agitação motora do recém-nascido em 30 casos $(50,0 \%)$,(Tabela 2$)$.

Em sete casos $(9,7 \%)$ a extubação ocorreu nas primeiras 48 horas de ventilação mecânica, em 17 casos $(23,6 \%)$ entre 2 a 7 dias; $8(11,1 \%)$ entre 8 a 14 dias; 15 (20,8\%) entre 15 e 30 dias; 18 (25,0\%) entre 31 e 60 dias e $9(12,5 \%)$ com mais de 60 dias. Metade dos casos ocorreu nos primeiros 21 dias do suporte ventilatório. Dos 72 casos de extubação, 29 (40,3\%) ocorreram no período da manhã (de 7:00h da manhã às 12:59h), 21 (29,2\%) à tarde (entre as 13:00h e 18:59h) e 22 (30,5\%) à noite (das 19:00h às 6:59h).

Reintubação foi necessária em 58 dos eventos (80,5\%). Em 20 deles (34,5\%) a reintubação foi imediata, nos demais casos $(65,5 \%)$ os recém-nascidos foram manejados, antes da reintubação, com oxigenoterapia por cateter nasal $(22,4 \%)$, ventilação com pressão positiva (VPP) $(17,2 \%)$, Continuous Positive Airway Pressure (CPAP) (12,0\%) e ventilação não invasiva (VNI) (19,0\%). Não se observou associação entre a necessidade de reintubação e o peso do recém-nascido $(p=0,22)$. Dos 14 eventos (19,5\%) em que não houve reintubação, 2 (14,3\%) foram tratados com oxigênio inalatório, 4 (28,6\%) com VPP, 4 (28,6\%) com CPAP e $4(28,6 \%)$ com VNI. Um recém-nascido, equivalente a $2,6 \%$ dos casos e $1,4 \%$ dos eventos de ENP foi a óbito, (Tabela 3). 
Tabela 1. Características dos 38 recém-nascidos em recém-nascidos internados em UTI Neonatal

\begin{tabular}{|c|c|}
\hline Características & $\mathrm{n}(\%)$ \\
\hline \multicolumn{2}{|l|}{ Sexo } \\
\hline $\mathrm{F}$ & $20(52,6 \%)$ \\
\hline$M$ & $18(47,4 \%)$ \\
\hline \multicolumn{2}{|l|}{ Peso } \\
\hline Menor 1000 & $13(34,2 \%)$ \\
\hline 1000 e 1499 & $7(18,5 \%)$ \\
\hline 1500 e 2499 & $4(10,5 \%)$ \\
\hline Maior que 2500 & $14(36,8 \%)$ \\
\hline \multirow{2}{*}{\multicolumn{2}{|c|}{$\begin{array}{l}\text { Diagnóstico } \\
\text { Idade Gestacional }\end{array}$}} \\
\hline & \\
\hline Prematuridade & $25(65,8 \%)$ \\
\hline A termo & $13(34,2 \%)$ \\
\hline Malformações congênitas & $11(28,9 \%)$ \\
\hline \multicolumn{2}{|l|}{ Cânula } \\
\hline Orotraqueal & $36(95,0 \%)$ \\
\hline Nasotraqueal & $2(5,0 \%)$ \\
\hline \multicolumn{2}{|l|}{ Número de ENP } \\
\hline 1 & $23(60,5 \%)$ \\
\hline 2 & $5(13,1 \%)$ \\
\hline$>2$ & $10(26,4 \%)$ \\
\hline
\end{tabular}

ENP: Extubação não planejada

Tabela 2. Características dos 72 eventos de extubação não planejada em recém-nascidos internados na UTI Neonatal

\begin{tabular}{cc}
\hline \multicolumn{1}{c|}{ Características } & $\mathrm{n}(\%)$ \\
\hline Sexo & $39(54,2 \%)$ \\
$\mathrm{F}$ & $33(45,8 \%)$ \\
Peso & $29(40,3 \%)$ \\
Menor 1000 & $10(13,9 \%)$ \\
1000 e 1499 & $6(8,3 \%)$ \\
1500 e 2499 & $27(37,5 \%)$ \\
Maior que 2500 & $72(100,0 \%)$ \\
Cânula & $0(0,0 \%)$ \\
Orotraqueal & \\
Nasotraqueal & $47(65,3 \%)$ \\
Diagnóstico & $22(34,7 \%)$ \\
Idade Gestacional & $24(33,3 \%)$ \\
Prematuridade & \\
A termo & \\
Malformações congênitas* & \\
Condição associada à ENP & $17(28,3 \%)$ \\
Troca/Fixação/Reposicionamento de CET & $9(15,0 \%)$ \\
Procedimentos de rotina & $4(6,7 \%)$ \\
Exame clínico & $30(50,0 \%)$ \\
Agitação psicomotora do RN &
\end{tabular}

ENP: Extubação não planejada 


\begin{tabular}{|c|c|}
\hline Características & $\mathrm{n}(\%)$ \\
\hline \multicolumn{2}{|c|}{ Dia em ventilação mecânica da ENP } \\
\hline 1 as $48 \mathrm{~h}$ & $7(9,7 \%)$ \\
\hline 2 a 7 dias & $17(23,6 \%)$ \\
\hline 8 a 14 dias & $8(11,1 \%)$ \\
\hline 15 a 30 dias & $15(20,8 \%)$ \\
\hline 31 a 60 dias & $18(25,0 \%)$ \\
\hline$>60$ dias & $9(12,5 \%)$ \\
\hline \multicolumn{2}{|l|}{ Período do dia da ENP } \\
\hline Manhã & $29(40,3 \%)$ \\
\hline Tarde & $21(29,2 \%)$ \\
\hline Noite & $22(30,5 \%)$ \\
\hline \multicolumn{2}{|l|}{ Manejo após a ENP } \\
\hline Reintubação* & $58(80,5 \%)$ \\
\hline Imediata & $20(34,5 \%)$ \\
\hline Após Oxigênio nasal & $13(22,4 \%)$ \\
\hline Após VPP & $10(17,2 \%)$ \\
\hline Após CPAP & $7(12,0 \%)$ \\
\hline Após VNI & $11(19,0 \%)$ \\
\hline Não reintubação & $14(19,5 \%)$ \\
\hline Oxigênio nasal & $2(14,2 \%)$ \\
\hline VPP & $4(28,6 \%)$ \\
\hline CPAP & $4(28,6 \%)$ \\
\hline VNI & $4(28,6 \%)$ \\
\hline Óbito & $1(1,4 \%)$ \\
\hline
\end{tabular}

\section{Discussão}

Entre as recomendações mais recentes ${ }^{\circledR}$, apontam que a taxa aceitável de ENP deve ser inferior a 2/100 pacientes intubados/dia, enquanto Merkel et al., , sugerem que deva e o ideal seja inferior a 1/100 e que a adoção de medidas de segurança pode reduzir esta taxa para 0,41/100, considerando ainda que é um incidente potencialmente evitávelę.9. Nessa amostra a prevalência encontrada foi de 4,6/100, e de 3,8/100 quando corrigida (excluindo os 2 recém-nascidos com malformações cervicais e alta recorrência de extubação não planejada), estando acima da taxa recomendada, mas dentro das variações reportadas na literatura. Não se observou diferença na frequência de ocorrência do incidente de acordo com o sexo, mas são poucos os estudos que avaliam este aspecto. Aydon, Zimmer e Sharp ${ }^{10}$ referiram taxa de ENP mais prevalente em recém-nascido do sexo masculino, com relação de 3:1. Outros autores também observaram maior prevalência no sexo masculino ${ }^{3}$.

A literatura aponta associação do peso de nascimento com ENP. Na revisão sistemática de 30 anos conduzida por Silva et al. $\frac{11}{}$, de 34.105 artigos potencialmente relevantes sobre o assunto, 15 foram selecionados de 192 estudos considerados inicialmente para avaliação mais detalhada. Entre eles Brown ${ }^{12}$ encontrou maior frequência de ENP em recém-nascido com peso inferior a $1.000 \mathrm{~g}$ (42\% versus $23 \%$ ), enquanto Harimoto et al. ${ }^{13}$ referiram que em $87 \%$ dos casos a extubação não planejada ocorreu entre recém-nascido com peso inferior a $2.500 \mathrm{~g}$, taxa semelhante à observada no presente estudo (62,5\%). Alguns autores, entretanto, não encontraram associação entre ENP e o peso de nascimento ou peso no momento da extubação ${ }^{14}$. Os eventos, aqui, distribuíram-se de forma bimodal: um pico em uma população de recém-nascido de muito baixo peso $(<1000 \mathrm{~g})(34,2 \%)$ e outro em recém-nascido com mais de $2.500 \mathrm{~g}(36,8 \%)$, refletindo, possivelmente, associação com prematuridade e sedação inadequada, como pode ser observado em outros estudos ${ }^{5,7}$. Verificou-se, ainda, eventos recorrentes (39,5\%), com risco 6 vezes maior em neonatos com peso inferior a 2.500g, evento este também descrito por Kleiber e Hummel 15 em $47 \%$ dos casos. 
O tempo de permanência em assistência ventilatória tem sido apontado como um dos principais fatores de risco para extubação não planejada $2,5,7$. A maior taxa de eventos situa-se em torno de 10,5 dias de ventilação mecânica 2,7 , Carvalho et al. $\frac{5}{\text { apon- }}$ taram que cada dia de VM aumenta o risco de ENP em $3 \%$. O procedimento de intubação nasotraqueal, embora considerado por alguns autores como mais seguro, é pouco utilizado devido às complicações como necrose das aletas nasais e hemorragia nasofaríngea. Da mesma forma, cânulas com balonete, utilizadas em UTI pediátricas e potencialmente mais seguras, não são utilizadas em neonatologia pelo risco de necrose traqueal16,17.

Entre os fatores que favorecem eventos de ENP em neonatos estão algumas características próprias do recém-nascido, como traqueia curta, imaturidade neurológica e face pequena. Outros fatores são a necessidade de manutenção do cuidado da fixação devido ao constante umedecimento, que pode ser causado tanto pela salivação quanto pelo uso de incubadoras umidificadas, e a facilidade de extubação, devido ao curto comprimento que define intubação efetiva, ou seja, milímetros de deslocamento podem determinar uma extubação $\mathrm{O}^{2-5}$.

A intubação de recém-nascido com malformações habitualmente é mais difícil, visto que podem determinar maior resistência à passagem do tubo endotraqueal. Outro fator determinante de maior prevalência de ENP é o maior número de procedimentos a que estes recém-nascido são submetidos, seja por medidas de investigação diagnóstica ou terapêutica. Os recém-nascidos que apresentavam higroma cístico e Síndrome de Prune Belly foram os que apresentaram maior recorrência de extubação (7 e 5 eventos, respectivamente) nesta amostra. Na literatura a malformação congênita é pouco tratada em análises de eventos ENP 18 .

Na revisão sistemática conduzida por Silva et al. ${ }^{11}$, as principais condições associadas à ENP foram agitação motora do recém-nascido (13-89\%), manuseio e fixação da cânula endotraqueal (17-30\% e 8-31\%, respectivamente) e procedimentos realizados à beira do leito (27,5-50\%). No presente estudo a agitação motora do recém-nascido esteve associada à ENP em metade dos casos, enquanto o manuseio da CET responsável por $28,3 \%$ dos casos.
Entre algumas das medidas de prevenção de ENP estão a sedação e medidas não farmacológicas. Não existem estudos adequadamente desenhados para avaliar o uso de sedação para prevenção da extubação. Os poucos estudos têm resultados controversos e, em UTI pediátricas e de adultos, a sedação não esteve associada à prevenção efetiva da ENP5 111,14,15,18-20. Além disso, é importante ressaltar que a agitação motora pode ser manifestação clínica de hipoxemia? Em estudos em que há uso rotineiro de sedação em recém-nascido sob ventilação mecânica, a taxa de agitação motora tende a ser inferior em situação de manipulação e procedimentos ${ }^{7}$, o que qualifica a sedação como um fator protetor para ENP. Contudo, implica em efeitos colaterais e, além de muitas classes não atuarem na analgesia, podem ser potencializadores da dor, exceto alguns opiáceos, que, além de interferir na agitação motora do neonato, atuam também na analgesia diante de estímulos dolorosos e procedimentos invasivos. Porém, esses medicamentos estão associados a depressão respiratória, retenção urinária, náusea, vômitos, diminuição do trânsito intestinal e dependência física ${ }^{21,22}$.

Como alternativa, a contenção é uma medida não farmacológica frequentemente utilizada. Na UTIN estudada, sedação farmacológica não é utilizada de rotina e os recém-nascido ficam acomodados em um ninho, formado por cueiros dobrados e posicionados de forma oval ao redor do paciente, provendo conforto e limitação de movimentos de risco para extubação. $\mathrm{Na}$ literatura, os resultados com medidas de contenção também não são consistentes, embora alguns autores recomendem ainda o uso de luvas nas mãos do RN, especialmente em recém-nascido com peso menor que $2.500 \mathrm{~g}$, evitando a retirada do tubo 12,17,20. A vigilância constante também é efetiva, desde que permite identificação precoce de agitação motora, podendo prevenir a ENP, visto que esta é menor quanto maior for a relação profissionais da área/paciente ${ }^{10}$.

A associação entre ENP e manuseio do neonato durante procedimentos de rotina é presente na maioria dos estudos. Incluem coleta de sangue, pesagem, mudança de decúbito, entre outros. Na UTI estudada, a menor taxa de extubação não planejada associada a procedimentos da equipe deve-se, possivelmente, à organização da equipe para o manuseio do RN com um ou mais profissionais, sendo eles médicos ou enfermeiros. A coleta de sangue, por exemplo, é habitualmente realizada em dois, em que o médico é 
responsável pelo procedimento de coleta e o auxiliar atua na contenção do paciente. A necessidade de pelo menos dois membros da equipe para realização de procedimentos em recém-nascido intubados é apontada por alguns autores e compõe uma das ações dos bundles de segurança no cuidado destes pacientes ${ }^{9,10}$.

Nos procedimentos envolvendo o manuseio da cânula endotraqueal, como troca de fixação, aspiração de secreções e reposicionamento, a taxa de ENP é maior devido ao manuseio específico do tubo endotraqueal, podendo ocasionar mobilidade inadvertida. Veldman et al. ${ }^{14}$ apontam a fixação inadequada da CET como uma das principais causas da extubação. Muitos métodos de fixação já foram propostos e incluem uso de fitas adesivas, clamps de cordão umbilical, suturas e aparatos de fixação da CET adaptados na cabeça do neonato. Lai et al. ${ }^{24}$, em revisão que incluiu todos os ensaios clínicos controlados de 1966 a 2013, concluíram que dado a heterogeneidade dos métodos utilizados, tanto de fixação como de desenhos dos estudos, há falta de evidência conclusiva sobre qual a melhor forma de fixação da CET em RN, e que a padronização do procedimento e experiência da equipe estão associadas a intubações mais seguras.

O uso de fitas adesivas é frequente em UTIN, mas com face pequena e pele sensível, deve-se ter cautela com o seu uso excessivo em recém-nascidos, limitando-se apenas aos centímetros necessários para fixação entre as rimas labiais $22-24$. No serviço estudado, o método de fixação utilizado é com fita adesiva cortada em forma de $\mathrm{H}$, sendo a CET posicionada centralmente, com a parte superior do $\mathrm{H}$ fixado na região nasolabial superior e a parte inferior na CET, sendo uma das hastes do $\mathrm{H}$ fixada em espiral e a outra de forma circular, rente ao lábio.

Silva et al. ${ }^{11}$ referiram que 39\% das ENP ocorrem nas primeiras 48 horas de ventilação mecânica, com 51,2\% dos casos ocorrendo nos primeiros 7 dias. Neste estudo estas frequências foram de $9,7 \%$ e $34,7 \%$, respectivamente. Ainda, foi observado que os eventos de ENP ocorreram com distribuição semelhantes nos períodos da manhã, tarde e noite, enquanto a revisão sistemática apontou $26,8 \%$ dos casos entre as 7 e 10 horas da manhã e $17 \%$ entre 16 e $19 h^{9,11}$. O período da manhã é considerado de maior risco pelo maior número de procedimentos realizados, como exames clínicos, laboratoriais e de imagem, procedimentos de higiene e bem-estar, procedimentos terapêuticos e ventilatórios, incluindo os cuidados com a CET 2 .

As complicações imediatas da ENP incluem, habitualmente, deterioração da função cardiorrespiratória, com bradicardia (39-46\%), ressuscitação cardiopulmonar (5-13\%), enquanto as tardias incluem aumento do tempo de ventilação mecânica (de 52 para 345h) e aumento do tempo de internação ( 9 para 51 dias), além do risco de necrose subglótica, pneumonia associada à VM e broncodisplasia pulmonar. A necessidade de reintubação estimada varia amplamente entre os estudos, indo de 3 a 100\% dos $\operatorname{casos}^{11}$ e ocorreu, nesta amostra, em $80 \%$ dos eventos, tendo sido imediata em 20 casos (34,5\%).

A modificação da cultura de segurança do paciente é essencial, voltada especialmente para a ciência e reconhecimento de que a ENP é um evento adverso evitável e de risco potencial para o RN. Aydon, Zimmer e Sharp ${ }^{10}$, em publicação recente, recomendam a implantação de um bundle de cuidados com a cânula que inclui: a) métodos padronizados de fixação da CET; b) guidelines de prevenção e manejo da extubação não planejada; c) documentação da posição da CET de acordo com o peso e idade do neonato; d) monitorização frequente e contínua da CET e das condições de sua fixação; e) divulgação sobre a incidência e fatores associados à ENP para o equipe; f) checklist documentado de procedimentos diante de ENP; g) envolvimento de 2 profissionais para manuseio de recém-nascido intubados; h) sessões de educação continuada sobre os efeitos da ENP e estratégias de prevenção.

Merkel et al.ำ relataram redução da taxa de ENP de 2,38 para 0,41/100 utilizando um bundle que incluiu, além das medidas anteriormente citadas, a colocação de avisos no leito sobre a posição da CET e de tarjas vermelhas identificando aqueles recém-nascido com maior risco de ENP, como recém-nascido com peso inferior a $1.000 \mathrm{~g}$, ENP prévia, história prévia de dificuldade de intubação, anomalias de face e/ou de vias aéreas que dificultam a intubação e condições respiratórias graves, onde o deslocamento inadvertido da CET pode trazer graves consequências para o recém-nascido. 
Embora seja considerado um evento adverso de elevada gravidade, a extubação não planejada não está associada com aumento da mortalidade 2,14 , tendo sido observado um caso de óbito na amostra aqui estudada. Sua morbidade, entretanto, não está bem estabelecida e os autores ressaltam a heterogeneidade dos estudos conduzidos sobre o assunto e a baixa documentação do evento, o que dificulta a análise e conhecimento de seu real impacto. O que se sabe, de fato, é que medidas simples de segurança possuem grande eficácia na redução da ENP e devem ser implementadas em UTIN para garantir o melhor cuidado para os recém-nascido em $\mathrm{VM}^{9-11}, \underline{23}$.

As limitações do estudo estão relacionadas a ausência dos dados referentes às idades gestacionais dos neonatos e os dias de uso da ventilação mecânica, estes dados não estavam contemplados no período da coleta dos dados, caracterizando uma limitação da ferramenta de coleta. Esse instrumento foi corrigido pela equipe do serviço, posteriormente ao período da pesquisa.

\section{Conclusão}

A prevalência de ENP na UTIN/CHC está superior a recomendação encontrada na literatura. A frequência de ENP foi maior em neonatos com peso menor que $2500 \mathrm{~g}$, em prematuros e naqueles que apresentavam malformações congênita associadas. Já a recorrência de ENP esteve associada principalmente ao peso do RN menor que $2500 \mathrm{~g}$ e à presença de malformações congênitas. As principais causas foram a agitação psicomotora do RN e à fixação inadequada da cânula endotraqueal. Dessa forma medidas simples de segurança relacionadas à cultura organizacional tem grande eficácia na redução da ENP e devem ser implementadas para garantir o melhor cuidado ao recém-nascido em ventilação mecânica.

\section{Contribuições das autoras}

Mattos MCL e Silva GA participaram do delineamento, busca, coleta dos dados, análise estatística dos dados da pesquisa, interpretação dos resultados e redação do artigo científico. Andreazza:MG participou do delineamento, coleta dos dados, análise estatística dos dados da pesquisa e interpretação dos resultados. Rodrigues FS e De Oliveira IC participaram da busca, análise estatística dos dados da pesquisa, interpretação dos resultados e redação do artigo científico. Cat MNL participou da concepção, delineamento, análise estatística dos dados da pesquisa, interpretação dos resultados.

\section{Conflitos de interesses}

Nenhum conflito financeiro, legal ou político envolvendo terceiros (governo, empresas e fundações privadas, etc.) foi declarado para nenhum aspecto do trabalho submetido (incluindo, mas não se limitando a subvenções e financiamentos, participação em conselho consultivo, desenho de estudo, preparação de manuscrito, análise estatística, etc.).

\section{Referências}

1. Carlo WA, Greenough A, Chatburn A. Advances in conventional mechanical ventilation. In: Boynton BR, Carlo WA, Jobe AH. New Therapies for Neonatal Respiratory Failure: a physiological approach. Cambridge: Cambridge University Press; 1994.

2. Oliveira PCR, Cabral LA, Schettino RC, Ribeiro SNS. Incidência e principais causas de extubação não planejada em unidade de terapia intensiva neonatal. Rev Bras Ter Intensiva. 2012;24(3):2305. doi: $10.1590 / 50103-507 \times 2012000300005$

3. Hermeto F, Martins BMR, Ramos JRM, Bhering CA, Sant'Anna GM. Incidência e principais fatores associados à falha de extubação em recém-nascidos com peso de nascimento \&lt; 1.250 gramas. J Pediatr. 2009;85(5):397-402.

4. Costa ACO, Schettino RC, Ferreira SC. Predictors of extubation failure and reintubation in newborn infants subjected to mechanical ventilation. Rev Bras Ter Intensiva. 2014;26(1):51-6. doi: 10.5935/0103-507X.20140008

5. Carvalho FL, Mezzacappa MA, Calil R. Incidence and risk factors of accidental extubation in a neonatal intensive care unit. J Pediatr. 2010;86(3):189-95. doi: 10.1590/S0021$\underline{75572010000300005}$ 
6. Nesbitt G, Guy KJ, König K. Unplanned Extubation and Subsequent Trial of Noninvasive Ventilation in the Neonatal Intensive Care Unit. Am J Perinatol. 2015;32(11):1059-63. doi: $\underline{10.1055 / \mathrm{s}-0035-1548536}$

7. Torres MIU, Pumarega MTM, Lara NRG, Bonís AM, García MEF, Alonso CRP. Frecuencia de extubaciones no programadas en una unidad de cuidados intensivos neonatales. Estudio antes y después. An Pediatr. 2014;80(5):304-9. doi: 10.1016/j. anpedi.2013.07.002

8. Ndakor SM, Nelson MU, Pinheiro JMB. Counting unplanned extubations: marked variation among neonatologists. J Perinatol. 2017;37(6):698-701. doi: 10.1038/jp.2016.273

9. Merkel L, Beers K, Lewis MM, Stauffer J, Mujsce DJ, Kresch MJ. Reducing Unplanned Extubations in the NICU. Pediatrics. 2014;133(5):1367-72. doi: 10.1542/peds.2013-3334

10. Aydon L, Zimmer M, Sharp M. Reporting the incidence of unplanned extubation in the neonatal intensive care unit. J Paediatr Child Health. 2018;54(7):784-7. doi: 10.1111/jpc.13850

11. Silva PSL, Reis ME, Aguiar VE, Fonseca MCM. Unplanned Extubation in the Neonatal ICU: A Systematic Review, Critical Appraisal, and Evidence-Based Recommendations. Respir Care. 2013;58(7):1237-45. doi: 10.4187/respcare.02164

12. Brown MS. Prevention of Accidental Extubation in Newborns. Am J Dis Child. 1988;142(11):1240-3. doi: $10.1001 /$ archpedi.1988.02150110118035

13. Horimoto $Y$, Tomie H, Hanzawa K, Nishida Y. Accidental extubations during respiratory management in a children's hospital. J Anesth.1991;5(2):142-5. doi: 10.1007/s0054010050142

14. Veldman A, Trautschold T, Weiss K, Fischer D, Bauer K. Characteristics and outcome of unplanned extubation in ventilated preterm and term newborns on a neonatal intensive care unit. Pediatr Anaesth. 2006;16(9):968-73. doi: 10.1111/j.14609592.2006.01902.x

15. Kleiber C, Hummel PA. Factors related to spontaneous endotracheal extubation in the neonate. Pediatr Nurs. 1989;15(4):347-51.
16. McMillan DD, Rademaker AW, Buchan KA, Reid A, Machin G, Sauve RS. Benefits of orotracheal and nasotracheal intubation in neonates requiring ventilatory assistance. Pediatrics. 1986;77(1):39-44

17. Conner GH, Maisels MJ. Orotracheal intubation in the newborn. Laryngoscope. 1977;87(1):87-91. doi: 10.1288/00005537-197701000-00010

18. Little LA, Koenig Jr JC, Newth CJ. Factors affecting accidental extubations in neonatal and pediatric intensive care patients. Crit Care Med. 1990;18(2):163-5. doi: 10.1097/00003246-199002000$\underline{00007}$

19. Bellu R, dWaal K, Zanini R. Opioids for neonates receiving mechanical ventilation: a systematic review and meta-analysis. Arch Dis Child Fetal Neonatal Ed. 2010;95(4):241-51. doi: 10.1136/ adc. 2008.150318

20. Franck LS, Vaughan B, Wallace J. Extubation and reintubation in the NICU: identifying opportunities to improve care. Pediatr Nurs. 1992;18(3):267-70.

21. Silva YP, Gomez RS, Máximo TA, Silva ACS. Sedation and analgesia in neonatology. Rev Bras Anestesiol. 2007;57(5):575-87. doi: 10.1590/S0034-70942007000500013

22. Dejonge $\mathrm{MH}$, White M. A comparison of two methods of oral endotracheal tube stabilization in neonatal patients. J Perinatol. 1998;18(6 Pt 1):463-5.

23. Loughead JL, Brennan RA, Dejuilio P, Camposeo V, Wengert J, Cooke D. Reducing Accidental Extubation in Neonates. Jt Comm J Qual Patient Saf. 2008;34(3):164-70. doi: 10.1016/s15537250(08)34019-7

24. Lai M, Inglis GDT, Hose K, Jardine LA, Davies MW. Methods for securing endotracheal tubes in newborn infants. Cochrane database Syst Rev. 2014;7. doi: 10.1002/14651858.CD007805

25. Volsko T, Chatburn R. Comparison of two methods for securing the endotracheal tube in neonates. Respir Care. 1997;42(3):288-91.

26. Santos VFR, Figueiredo AEPL. Intervenção e atividades propostas para o diagnóstico de enfermagem - Ventilação espontânea prejudicada. ACTA Paul Enferm. 2010;23(6):824-30. doi: 10.1590/S0103-21002010000600017 